Research letter

\title{
Correlation of functional disorders in cerebral blood flow with cognitive style of freshmen students
}

\author{
Elena S. Olenko ${ }^{1}$, Ekaterina V. Fomina ${ }^{1}$, Anna I. Kodochigova ${ }^{1}$, Anton R. Kiselev ${ }^{2}$, Vyacheslav F. Kirichuk ${ }^{1}$ \\ ${ }^{1}$ Saratov State Medical University, Saratov, Russia \\ 2 National Medical Research Center for Therapy and Preventive Medicine, Moscow, Russia
}

Received 21 July 2021, Revised 9 August 2021, Accepted 23 August 2021

(C) 2021, Russian Open Medical Journal

\begin{abstract}
In 97 healthy male freshmen students, we studied the parameters of arterial blood flow intensity (index of vascular resistance RI, amplitude-frequency index AFI), venous outflow (venous outflow index VOI), and tone and elasticity of cerebral vessels (diastolic index DIA, dicrotic index DIC, maximum blood velocity of fast filling in the artery Vmax, average blood velocity of slow filling in the artery Vav). Separately, we assessed the effectiveness of such mental operations as classification and analysis and studied the cognitive styles. In $35.9 \%$ of male freshmen, we revealed the functional dystonia of cerebral vessels with a pronounced inter-lacunar redistribution of blood flow to the region of internal carotid arteries. Their cognitive style had low rigidity, flexible control, strong cognitive automation, and low information processing speed. The cognitive style in freshmen without dystonic disorders was characterized by a balance of rigidity and flexibility of thinking, with a medium information processing speed.
\end{abstract}

Keywords: freshmen students, functional dystonia of cerebral blood vessels, cognitive style.

Cite as Olenko ES, Fomina EV, Kodochigova Al, Kiselev AR, Kirichuk VF. Correlation of functional disorders in cerebral blood flow with cognitive style of freshmen students. Russian Open Medical Journal 2021; 10: e0308.

Correspondence to Elena S. Olenko. Email: olenco@mail.ru.

\section{Introduction}

At present, the structure of educational process makes high demands on students with continuous stress of their memory, attention and thought processes [1, 2, 3, 4]. The problem of improving cognitive functions is relevant not only when they deteriorate due to some organic disease, but also in healthy people experiencing high intellectual load in the process of starting educational activity [5]. Studies of the severity of cognitive deficits in young people are scarce. According to the definition, cognitive functions provide an individual's interaction with the environment [6]. At the same time, the integration of a person into the environment is implemented by the adaptive processes of autonomic regulation, which directly ensures the reactivity of the organism on the basis of information obtained by means of cognition [6, 7].

We are aware that cognitive impairments in youth are almost always comorbid and have a dystonic vegetative nature $[8,9]$. It was previously shown that the optimal level of providing cognitive functions in virtually healthy young people is achieved by a relative balance of sympathetic and parasympathetic influences, and an imbalance of autonomic regulation of the body (with a predominance of the vagus nerve) is accompanied by an increase in the severity of cognitive impairment [9]. In this regard, it is of particular importance to study the relationship between functional dystonic disorders of cerebral blood flow and cognitive styles in freshmen students. Familiarity with these relationships could help increasing the productivity of educational activities of school and university students, opening up opportunities for developing sound methods of improving their effectiveness.
The objective of our study involved comparative investigation of cognitive styles vs. cerebral blood flow condition in healthy male freshmen.

\section{Material and Methods}

Our study included 97 male freshmen students with an average age of $19.0(18.0,19.0)$ years old (data presented as median, lower and upper quartiles). The research was conducted in accordance with the ethical and legal standards set out in the Declaration of Helsinki by the World Medical Association in 1964, with subsequent amendments in 2000 and 2008, and was properly approved by the Biomedical Ethics Committee at Saratov State Medical University, Protocol No. 3 of April 6, 2018. Voluntary informed consent was signed by all prospective study subjects. All research procedures were standardized. The equipment and software were certified.

The study of cerebral hemodynamics was carried out using the 6-channel multifunctional computer rheograph Rheo-Spectrum-3, manufactured by NeuroSoft LLC (Ivanovo, Russia), which allowed recording 4-channel bipolar rheoencephalography (REG) with an automated assessment of indicators of the arterial blood flow intensity (index of vascular resistance [RI], amplitude-frequency index $[\mathrm{AFI}]$ ); of the arterial tone and elasticity (diastolic index [DIA], dicrotic index [DIC], maximum blood velocity of fast filling in the artery [Vmax], average blood velocity of slow filling in the artery [Vav]); and of venous hemodynamics (venous outflow index [VOI]). The difference in blood filling (RI) was used to assess coefficient of asymmetry (CA) of blood filling among different 
blood vessel regions and brain hemispheres: if CA was $7 \%$ or less, then there was no significant asymmetry of blood filling; with CA value ranging 8 to $14 \%$, the asymmetry of blood filling was characterized as small; CA from 15 to $25 \%$ indicated the presence of moderate blood filling asymmetry; at CA values of $26 \%$ or more, it was regarded substantial [10].

To identify dystonic disorders, we employed a functional test with changes in the head position (turns to the right, left, flexion and extension). In each position, the recording was carried out for 30 seconds. In persons with impaired REG during background recording, to distinguish between functional and organic changes in the vascular wall, after a 15-minute rest, a pharmacological test with nitroglycerin was performed [11-13].

To assess the effectiveness of mental operations of classification and analysis and the ability to highlight the essential features of an object, the Elimination by Aspects method was used [14]. In order to investigate cognitive styles (rigidity/flexibility of the cognitive process), the Stroop test was used $[15,16]$.

Statistical analysis was performed using Statistica 6.1 software package (StatSoft, USA). Quantitative data are presented in this article as median with lower and upper quartiles - Me (LQ, UQ). Methods of nonparametric statistics based on Mann-Whitney test were used. To identify the magnitude of statistical dependence of the distribution of the variables' values, Spearman's rank correlation coefficients were calculated. Statistical significance was stated at $p$-values $<0.05$

\section{Results}

The analysis of the REG results during a functional test with a change in the head position (turns to the right, left, flexion and extension) of each male freshman student has revealed dystonic disorders in the form of decreases in arterial inflow and venous outflow, as well as a reduction in the tone of medium and small arteries in the internal carotid region, and even more so in the vertebrobasilar arteries $(p<0.05)$ in $35.9 \% \quad(n=37)$ of healthy freshmen students.

On the basis of this finding, and for clarity of the cognitive process study, all students were split among two groups: G1 (comparison group, $\mathrm{n}=60$, without dystonic disorders) and $\mathrm{G} 2$ (experimental group, $n=37$, with dystonic disorders of cerebral blood flow). To provide differential diagnosis of the functional and organic nature of revealed dystonic changes in male students in G2, a test with nitroglycerin was used (Table 1), which demonstrated a significant increase in the vascular resistance index in all leads ( $p=0.04$ ); RI from 50.7 to $65.5 \%$ ) in the region of the vertebral and internal carotid arteries, compared with a background recording, which indicated maintaining elastic properties of the vascular wall and the functional nature of revealed dystonia.

When analyzing the mean values from the background REG recording, we established that, in G1 men, the indicators of arterial blood flow intensity ( $\mathrm{RI}$ and $\mathrm{AFI}$ ) in the right and left hemispheres of all lacunae were below the norm, whereas the values of the tone of large, medium and small arteries, peripheral vascular resistance and venous outflow were within the age norm (Table 2). In G2 men, with a background recording in a state of physiological rest, indicators of arterial blood flow intensity (RI, $\mathrm{AFI}$ ), the tone of large, medium and small arteries (Vmax and Vav), as well as the indicator of the tone of arterioles and the state of peripheral vascular resistance (DIA) in the vertebrobasilar regions on both sides were below the age norm (Table 2).

Analysis of the coefficient of asymmetry (CA) of inter-lacunar and interhemispheric redistribution of cerebral blood flow in freshmen students with dystonic disorders (G2) revealed a significant increase in inter-lacunar asymmetry of blood flow in both hemispheres with increased cerebral hemodynamics in the region of internal carotid arteries (CA on the right $=30.6 \%$, CA on the left $=48.3 \%$ ).

The cognitive process study demonstrated that freshmen in both groups had high levels of mental operations of classification and analysis (above 8 points in G1 and G2) and an ability to highlight the essential features of objects, make comparisons and generalizations. According to the Stroop test, task execution time, the coefficient of interference (Cint) and the number of mistakes differed significantly among $G 1$ and $G 2(p=0.04)$. The coefficient of imagery-verbality (Civ) in all students corresponded to the sensory perceptual information processing and the prevalence of imagery thinking (Table 3).

To clarify the relationship between the indicators of cerebral blood flow and cognitive process, the Spearman rank correlation analysis was applied, which did not reveal any dependencies among students in $\mathrm{G} 1(\mathrm{r}=0.29 ; \mathrm{p}=0.07)$. Freshmen with dystonic disorders (G2) exhibited a moderate feedback between RI (Fms) and Cint ( $r=-0.39, p=0.04)$, as well as RI (Fmd) and Cint $(r=-0.37$, $p=0.03$ ); they also demonstrated direct moderate relationship between Vav (Fms) and task execution time $(T, s)(r=0.52 p=0.02)$, and $\operatorname{Vav}(F m d)$ and task execution time $(T, s)(r=0.44 p=0.03)$.

\section{Discussion}

Functional dystonia of various regions of the peripheral vascular bed in youth is a frequent phenomenon of autonomic imbalance $[17,18]$. In the present study, it was shown that all healthy male freshmen at rest had a norm-resistant type of cerebral hemodynamics ( $D I C=40-70 \%$ ). However, the test with head turns and the subsequent nitroglycerin test revealed dystonic functional disorders in all lacunae in $35.9 \%(n=37)$ of virtually healthy freshmen students. In students without dystonia in a state of physiological rest, we observed a reduction in the intensity of arterial blood flow in the regions of the internal carotid and vertebrobasilar arteries on both sides with age-appropriate values of arteriole tone and the state of peripheral vascular resistance. In male students with functional dystonic phenomena, with background recording in a state of physiological rest, there was a pronounced inter-lacunar asymmetry of blood flow with its increase in the region of the internal carotid arteries feeding the prefrontal brain cortex. The latter is known to be responsible for higher nervous activity and cognitive processes [19].

Table 1. Test with nitroglycerin in G2 freshmen

\begin{tabular}{lcccc} 
REG & & REG indicators & & \\
leads & $\begin{array}{c}\text { RI (conditional units) } \\
\text { Background recording } \\
(\mathrm{n}=37)\end{array}$ & $\begin{array}{c}\text { RI (conditional units) } \\
\text { Test with nitroglycerin } \\
(\mathrm{n}=37)\end{array}$ & $\begin{array}{c}\mathrm{P} \\
\%\end{array}$ & $\begin{array}{c}\text { Increase } \\
\text { in RI }\end{array}$ \\
\hline Fms & $1.29(0.84,1.35)$ & $1.98(1.65,2.19)$ & 0.04 & 53.4 \\
Oms & $0.87(0.56,0.95)$ & $1.44(1.23,1.84)$ & 0.02 & 65.5 \\
Fmd & $1.28(0.92,1.34)$ & $1.93(1.68,2.21)$ & 0.03 & 50.7 \\
Omd & $0.98(0.55,1.33)$ & $1.52(1.26,1.86)$ & 0.04 & 55.1 \\
\hline
\end{tabular}

Legends for leads and REG indicators are presented in Table 2. Data presented as median with lower and upper quartiles - Me (LQ, UQ). 
Table 2. Average REG indices with background recording in freshmen students

\begin{tabular}{|c|c|c|c|c|c|c|c|}
\hline REG indicators & $R l$, conditional units & AFI, conditional units & $D I C, \%$ & $D I A, \%$ & Vmax, Ohm/s & Vav, Ohm/s & VOI, \% \\
\hline REG leads & \multicolumn{7}{|c|}{ G1 males $(n=60)$ Background recording at rest at a heart rate of $70.0(66.0 ; 76.0)$ beats per minute } \\
\hline Fms & $0.98 \downarrow(0.83,1.31)$ & $1.23 \downarrow(1.04,1.57)$ & $51.50(42.00,59.70)$ & $59.00(49.25,66.00)$ & $1.63(1.37,1.91)$ & $0.90(0.73,1.04)$ & $5.00(4.00,10.00)$ \\
\hline Oms & $0.88 \downarrow(0.66,1.15)$ & $1.09 \downarrow(0.78,1.32)$ & $64.40(48.40,76.00)$ & $.00(69.25,87.00)$ & $2,1.75)$ & 0.7 & 7.00 \\
\hline Fmd & $1.13 \downarrow(0.90,1.42)$ & $1.14 \downarrow(0.70,1.80)$ & $50.87(38.02,57.75)$ & $60.00(49.20,66.70)$ & $1.88(1.50,2.35)$ & $1.10(0.83,1.44)$ & $8.00(3.00,16.00)$ \\
\hline Omd & $0.95 \downarrow(0.71,1.30)$ & $1.13 \downarrow(0.91,1.61)$ & $58.00(47.97,66.50)$ & $74.50(67.00,87.75)$ & $1.59(1.23,2.06)$ & $0.84(0.69,1.06)$ & $10.00(5.00,17.00)$ \\
\hline REG & \multicolumn{7}{|c|}{ G2 males ( $n=37)$ Background recording at rest at a heart rate of $75.5(68.75,85.0)$ beats per minute } \\
\hline Fms & 1.35) & $1.37(0.98,1.61)$ & $50.00(44.80,64.50)$ & $58.00(49.70,64.50)$ & $1.47(1.16,2.03)$ & $0.85(0.66,1.09)$ & 7.0 \\
\hline Oms & $0.87 \downarrow(0.56,0.95)$ & $0.90 \downarrow(1.74,1.23)$ & $54.50 \downarrow(42.00,64.50)$ & $80.00(66.00,90.00)$ & $1.15 \downarrow(0.81,1.47)$ & $0.66 \downarrow(0.46,0.87)$ & $11.00(5.00,20.00)$ \\
\hline Fmd & $1.28(0.92,1.34)$ & $1.49(1.21,1.86)$ & $52.00(38.00,62.50)$ & $58.00(51.50,66.50)$ & $1.98(1.32,2.28)$ & $1.17(0.79,1.27)$ & $7.00(5.50,18.50)$ \\
\hline Omd & $0.98 \downarrow(0.55,1.33)$ & $1.18 \downarrow(0.77,1.71)$ & $52.00 \downarrow(44.60,70.00)$ & $75.00(61.00,88.05)$ & $1.42 \downarrow(0.96,1.95)$ & $0.75 \downarrow(0.53,0.98)$ & $6.50(13.00,19.00)$ \\
\hline
\end{tabular}

$\mathrm{RI}$, index of vascular resistance (conditional units); AFI, amplitude-frequency index (Ohm/s); DIC, dicrotic index (\%); DIA, diastolic index (\%); Vmax, maximum blood velocity of fast filling in the artery (Ohm/s); Vav, average blood velocity of slow filling in the artery (Ohm/s); VOI, an indicator of venous outflow (\%); Fms, frontal-mastoid lead on the left; Fmd, frontal-mastoid lead on the right; Oms, occipitomastoid lead on the left; Omd, occipitomastoid lead on the right. Data presented as median with lower and upper quartiles - Me (LQ, UQ).

Table 3. Results of studying the cognitive function in freshmen students: Me $(25 \%, 75 \%)$

\begin{tabular}{|c|c|c|}
\hline Investigated indicators (units) & G1 $(n=60)$ & G2 (n=37) \\
\hline Effectiveness of mental operations of classification and analysis via the Elimination by Aspects method (points) & $14.0(12.0,15.0)$ & $12.5(11.0,14.0)$ \\
\hline \multicolumn{3}{|l|}{ Indicators and values of the Stroop test } \\
\hline Task execution time $(\mathrm{T}, \mathrm{s})$ & $360.8(310.5,396.4)$ & $480.7(404.6,5107) *$ \\
\hline Coefficient of imagery-verbality (Civ, points) & $1.0(1.0,1.1)$ & $1.1(1.0,1.2)$ \\
\hline Number of mistakes (units) & $2.5(2.0,3.0)$ & $5.5(4.0,7.0) *$ \\
\hline
\end{tabular}

* $\mathrm{p} \leq 0.04$ for G2 vs. G1. Data presented as median with lower and upper quartiles - Me (LQ, UQ).

Earlier studies revealed that functional changes in cerebral blood flow could contribute to the onset of cognitive dissonance in people of different ages [20, 21]. Our research established that all freshmen, in general, had high levels of mental operations of classification and analysis. However, in students with dystonic disorders, there was a tendency to a reduction in the ability to distinguish essential features of an object, along with making comparisons and generalizations (Table 3 ). The results of studying cognitive styles using the Stroop test implied that, in individuals without dystonic disorders, the processes of rigidity and flexibility of thinking were well balanced, and the speed of information processing corresponded to medium values: $\operatorname{Cint}=1.5(1.2,1.7)$ bits per second. Students with dystonic disorders had significantly lower coefficient of interference values - Cint $=0.7(0.6,0.9)$ - with an increase in task execution time to $480.7(404.6,5107) \mathrm{s}$ and the number of mistakes made $5.5(4.0,7.0)$ units $(p \leq 0.04)$. Interference in that case was the result of a conflict between verbal and sensory-perceptual functions: the subject was supposed to ignore (suppress) the meaning of the word and, instead, name the color that he saw [22]. Low interference indicated the ability to inhibit verbal functions that were stronger by nature for the sake of color perception, that is, it confirmed low rigidity and high flexibility of the cognitive process with strong automation of cognitive functions [23]. The coefficient of imageryverbality (Civ) in all freshmen students corresponded to the sensory-perceptual way of processing information and the prevalence of imagery over verbality.

It is often difficult to determine the relationship between indicators of cerebral blood flow intensity and cognitive processes in healthy individuals [24], while in patients with symptomatic atherosclerotic lesions of major cranial arteries, cognitive impairment occurs in the vast majority of cases $[25,26]$. In addition to many organic causes, the state of autonomic balance affects the cognitive function of a healthy person, especially in youth [27]. The relationship between the quality of memorization process and the emotional significance of information in children with autonomic dysfunction syndrome was previously described [8]. That is why it was interesting to search for relationships between indicators of cerebral blood flow (identified via REG) and cognitive process in healthy male freshmen students. Our data demonstrated that there was no significant relationship in students without signs of cerebral dystonia. However, in individuals with functional dystonic changes, inverse and direct relationships of a moderate strength were revealed: an increase in the intensity of blood flow in the region of internal carotid arteries on both sides [RI (Fms), RI (Fmd)] correlated with low rigidity and high flexibility of the cognitive process with strong automation of cognitive functions (Cint); an increase in the tone of medium and small arteries in the region of internal carotid arteries [Vav (Fms), Vav (Fmd)] was associated with an increase in the time required for executing the Stroop test task $(T, s)$. This finding is interesting because the functional nature of cerebral vascular dystonia with a redistribution of hemodynamic flows to the region of internal carotid arteries may be associated with a conscious need to enhance mental activity and, hence, is just a manifestation of an individual cognitive style, but this hypothesis requires further study.

\section{Conclusion}

In presented study, according to the results of rheoencephalography, in $35.9 \%$ of male freshmen students, functional dystonia of the cerebral vessels with a pronounced inter-lacunar redistribution of blood flow to the region of the internal carotid arteries was revealed. Their cognitive style had low rigidity, flexible control, strong cognitive automation, and low information processing speed. In freshmen without dystonic disorders, the cognitive style was characterized by a balance of rigidity and flexibility of thinking, with a medium speed of information processing. 


\section{Conflict of interest}

We declare no conflicts of interest.

\section{References}

1. Malenova AYu, Malenov AA. Features of coping with information stress at school age. Social'naya rabota: sovremennye problemy $i$ tehnologii 2020; (1): 132-144. https://www.elibrary.ru/item.asp?id=42358154.

2. Goryachuk IV, Finogenko El. Prevention and correction of maladaptive conditions of students formed under the influence of information stress. Young Researchers' Journal of ISTU 2012; (2): 13. Russian. https://www.elibrary.ru/item.asp?id=19071797.

3. Zotova OM, Zotov VV. Information overload as a stress factor among university students. Humans and their health 2015; (4): 108-115. Russian. https://www.elibrary.ru/item.asp?id=25380172.

4. Mukhamedyanova AR, Shiryaev VB. Severity of information stress and ways of its optimization. Symbol of Science 2017; 3(3): 203-206. Russian. https://elibrary.ru/item.asp?id=28925890.

5. Poltorak MS, Grom VL, Sarchuk EV. Assessment of level of stress resistance students of medical university. Juvenis Scientia 2019; (4): 47. Russian. https://elibrary.ru/item.asp?id=37383191.

6. Chaynikov PN, Cherkasova VG, Kulesh AM. Cognitive functions and mental performance of team sports athletes. Sports medicine: research and practice 2017; 7(1): 79-85. Russian. https://doi.org/10.17238/ISSN2223-2524.2017.1.79.

7. Kravtsova EYu, Obukhov AS. The condition of adaptationcompensatory systems of children with organic brain injuries during academic year according to the data of variational cardiointervalography. Medical Almanac 2012; (5(24)): 119-121. Russian. https://elibrary.ru/item.asp?id=18141758

8. Antropov ES, Cherkasova VG, Muravyev SV, Krylova IV. The comparative characteristic of condition of bone-muscular and autonomic nervous systems of mountain climbers of childhood and adolescence ages, depending on the level of sportsmanship. The Russian Journal of Physical Education and Sport 2016; 11(4): 195-202. Russian. https://doi.org/10.14526/01_1111_167.

9. Wetzler MV, Cherkasova VG, Muravyev SV, Kulesh AM, Chainikov PN, Solomatina NV, et al. Features and correlation of cognitive function and autonomic regulation in healthy young adults. Postgraduate Doctor 2018; 87(2): 11-18. Russian. https://elibrary.ru/item.asp?id=32611606.

10. Yarullin HH. Clinical Rheoencephalography. Moscow, Russia: Medicine. 1983; 276 p. Russian.

11. Ronkin MA, Ivanov LB. Rheography in Clinical Practice. Moscow, Russia: MBN. 1997; 403 p. Russian.

12. Lüscher TF, Noll G. The pathogenesis of cardiovascular disease: Role of the endothelium as a target and mediator. Atherosclerosis 1995; 118 Suppl: S81-S90. https://pubmed.ncbi.nlm.nih.gov/8821468/.

13. Sugawara J, Maeda S, Otsuki T, Tanabe T, Ajisaka R, Matsuda M. Effects of nitric oxide synthase inhibitor on decrease in peripheral arterial stiffness with acute low-intensity aerobic exercise. Am J Physiol Heart Circ Physiol 2004; 287(6): H2666- H2669. https://doi.org/10.1152/ajpheart.00077.2004.

14. Safonova SH. Exclusion of concepts method. In: Pathopsychology: Reader. Compiled by NL Belopolskaya. 2nd ed. Moscow, Russia: Kogito-Tsentr. 2000: 209-212. Russian. http://pedlib.ru/Books/3/0021/3 0021-145.shtml.

15. Panina NG, Isupov IB, Ushanov GA. Cerebral circulation as an indicator of physical performance in athletes. Aktualnye voprosy nauki 2015; (18): 176-178. Russian. https://elibrary.ru/item.asp?id=23561164.

16. Stroop JR. Studies of interference in serial verbal reactions. Journal of

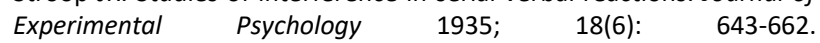
https://psycnet.apa.org/doi/10.1037/h0054651.
17. Isupov IB. Systemic Analysis of Human Cerebral Circulation. Volgograd, Russia: Peremena. 2001; 139 p. Russian.

18. Isupov IB. Blood circulation in the brain and forearm region of young men performing physical work of moderate intensity. Grani poznanija 2010; (3(8)): 36-38. Russian. https://elibrary.ru/item.asp?id=17910132.

19. Machinskaya RI. The Brain Executive Systems. Zh Vyssh Nerv Deiat I P Pavlova 2015; 65(1): 33-60. Russian. https://doi.org/10.7868/S0044467715010086.

20. Sokolova LP. Features of the cerebral blood supply in cases of predemential cognitive impairments of various origin. Modern problems of science and education 2011; (4): 8. Russian. https://elibrary.ru/item.asp?id=16985677.

21. Desmond DW. The neuropsychology of vascular cognitive impairment: Is there a specific cognitive deficit? J Neurol Sci 2004; 226(1-2): 3-7. https://doi.org/10.1016/j.jns.2004.09.002.

22. Sysoeva TA. Theoretical analysis of emotional stroop effect mechanisms. Psychology. Journal of the Higher School of Economics 2014; 11(1): 49-65. Russian. https://elibrary.ru/item.asp?id=23593837.

23. Allakhverdov VM, Allakhverdov MV. Stroop effect: interference as a logic paradox. Vestnik of Saint Petersburg University. Seria 16. Psychology. Education 2014; (4): 90-102. Russian. https://elibrary.ru/item.asp?id=22574877.

24. Schurov VA. Cerebral blood flow velocity and human efficiency. Russian Journal of Biomechanics 2016; 20(4): 352-357. Russian. https://doi.org/10.15593/RZhBiomeh/2016.4.07.

25. Pospelova ML, Zaytsev DE, Lepekhina AS, Efimtsev AY, Alekseeva TM, Trufanov GE. Cognitive lesion in patients with asymptomic carotid stenoses more than $70 \%$ - indication for surgical treatment? Modern problems of science and education 2019; 5: 116. Russian. https://elibrary.ru/item.asp?id=41258214.

26. Sweetow RW. Screening for cognitive disorders in older adults in the audiology clinic. Audiology Today 2015; 27(4): 38-43.

27. Kuznetsova EA. Cognitive features of adolescents with somatoform autonomic dysfunction. Bulletin of the South Ural State University. Series "Psychology" 2016; 9(1): 84-92. Russian. https://doi.org/10.14529/psy160109.

\section{Authors:}

Elena S. Olenko - MD, DSc of the Department of Normal Physiology, Saratov State Medical University, Saratov, Russia. https://orcid.org/00000003-1573-0623.

Ekaterina V. Fomina - Assistant of the Department of Normal Physiology, Saratov State Medical University, Saratov, Russia. https://orcid.org/00000002-3641-6476.

Anna I. Kodochigova - MD, DSc Professor of the Department of Propaedeutics of Internal Diseases Saratov State Medical University, Saratov, Russia. https://orcid.org/0000-0002-4717-5671.

Anton R. Kiselev - MD, DSc, Head of Coordinating Center for Fundamental Research, National Medical Research Center for Therapy and Preventive Medicine, Moscow, Russia. https://orcid.org/0000-0003-3967-3950.

Vyacheslav F. Kirichuk - MD, DSc, Professor of the Department of Normal Physiology, Saratov State Medical University, Saratov, Russia. https://orcid.org/0000-0002-5853-7316. 